\title{
Relaciones ecológicas entre frutos hospederos, moscas frugívoras y parasitoides en un fragmento de bosque seco tropical
}

\author{
Ecologic relationships among host fruits, frugivorous flies and parasitoids in \\ a fragment of tropical dry forest
}

Jeferson Saavedra-Díaz ; Pedro Edgar Galeano-Olaya ${ }^{2}$; Nelson Augusto Canal D. ${ }^{3}$

1. Investigador Asociado, Ingeniero Agrónomo. Grupo de Investigación en Moscas de las Frutas. Universidad del Tolima. Ibagué. Colombia. jsaavedrad@ut.edu.co.

2. Profesional Universitario, Administrador Agropecuario. Grupo de Investigación en Moscas de las Frutas. Universidad del Tolima. Ibagué. Colombia. pegalean@ut.edu.co.

3. Profesor Asociado, Ph.D. en Entomología. Grupo de Investigación en Moscas de las Frutas. Universidad del Tolima. Ibagué. Colombia.nacanal@ut.edu.co.

Citar: SAAVEDRA-DÍAZ, J.; GALEANO-OLAYA, P.; CANAL, N. 2017. Relaciones ecológicas entre frutos hospederos, moscas frugívoras y parasitoides en un fragmento de bosque seco tropical. Rev. Cienc. Agr. 34(1): 32 - 49. doi: http:// dx.doi.org/10.22267/rcia.173401.61.

Recibido: Septiembre 13 de 2016. Aceptado: Noviembre 11 de 2016.

\section{RESUMEN}

Las moscas de las frutas (Tephritoidea: Tephritidae y Lonchaeidae) son una de las principales plagas de la fruticultura a nivel mundial. El conocimiento de las interacciones que presentan las moscas frugívoras con sus plantas hospederas y parasitoides es necesario para el desarrollo de programas de manejo integrado de estas plagas. El objetivo de este trabajo fue describir las relaciones tritróficas (plantas hospederas, moscas frugívoras y parasitoides) en un parche de bosque seco tropical en el Centro Universitario Regional del Norte del municipio de Armero-Guayabal, Tolima. Se colectaron frutos de 35 especies de plantas. Los frutos se transportaron al laboratorio y se dispusieron en forma individual para la posterior pupación y emergencia de moscas y/o parasitoides. Se registraron 2.940 larvas en frutos de 15 especies de plantas. De estas larvas se obtuvieron adultos de seis especies de Tephritidae (cinco de Anastrepha y Toxotrypana curvicauda) y ocho de Lonchaeidae (seis de Neosilba y dos de Lonchaea). Los tefrítidos fueron los más abundantes y causaron los mayores porcentajes de daño (0-83,31\%) e índices de infestación (0-10,32 larvas/fruto y 0-463,37 larvas/kg frutos), y los lonquéidos fueron más polífagos con 12 plantas hospederas. Ambas familias compartieron hospederos y se presentaron cohabitando un mismo fruto. Cuando se 
encuentran en simpatría las especies de Anastrepha tienden a no compartir sus plantas hospederas o un mismo fruto. Las especies de Neosilba si comparten hospederos y frutos. Se encontraron cinco especies de parasitoides. Nuevos registros y asociaciones de plantas hospederas, moscas frugívoras y parasitoides se presentan para Colombia.

Palabras clave: Tephritidae, Lonchaeidae, parasitismo, biodiversidad, ecología.

\begin{abstract}
Fruit flies (Tephritoidea: Tephritidae and Lonchaeidae) are one of the main worldwide pests of fruits. The knowledge of the interactions among frugivorous flies with their host plants and parasitoids is necessary to develop integrated pest management programs. The aim of this study was to describe the tritrophics relationship (host plants, frugivorous flies and parasitoids) in a patch of dry tropical forest in the Centro Universitario Regional del Norte, Armero-Guayabal, Tolima, Colombia. Fruits of 35 different plant species were collected. The fruits were transported to the laboratory and were disposed individually for subsequent pupation and the emergence of flies and / or parasitoids. 2,940 larvae were recovered from fruits of 15 plant species; adults of six species of Tephritidae (five Anastrepha and Toxotrypana curvicauda) and eight of Lonchaeidae (six Neosilba and two Lonchaea) were obtained. Tephritids were the most abundant and caused the highest percentages of damage ( 0 to $83.31 \%$ ) and infestation rates ( 0 to 10.32 larvae / fruit and 0 to 463.37 larvae/kg fruit), and Lonchaeids were the most polyphagous, infesting 12 host plants. Both families shared hosts. Sympatric species of Anastrepha neither share their host plants nor cohabit the same fruit; Neosilba species share hosts and fruits. Five species of parasitoids were collected. New records and associations of host plants, frugivorous flies and parasitoids are presented for Colombia.
\end{abstract}

Key words: Tephritidae, Lonchaeidae, parasitism, biodiversity, ecology.

\section{INTRODUCCIÓN}

La fruticultura es uno de los sectores más importantes para el crecimiento de la agricultura colombiana (Miranda, 2011). Entre las plagas de mayor importancia para la fruticultura se encuentran las moscas frugívoras (Diptera: Tephritoidea), representadas por las familias Tephritidae y Lonchaeidae (UchôaFernandes y Zucchi 1999; Castañeda et al., 2010; Medina y Kondo, 2012; Uchôa, 2012; Wyckhuys et al., 2012; MacGowan y Okamoto, 2013). Las moscas frugívoras limitan la comercialización debido al daño directo que ocasionan y a las restricciones cuarentenarias de los países importadores (Castañeda et al., 2010; Miranda, 2011; Sarmiento et al., 2012; Ruiz-Hurtado et al., 2013; Gonçalves et al., 2016). En Colombia, el manejo de estas plagas se ha enfocado principalmente al uso de trampas, cebos tóxi- cos y aplicaciones químicas (Quintero et al., 2012; Santamaría et al., 2014); sin embargo, otras alternativas más amigables se han utilizado para reducir el uso indiscriminado de agroquímicos (Aluja et al., 2009; Quintero et al., 2012; Garcia y Ricalde, 2013; Gonçalves et al., 2016).

Para el desarrollo e implementación de programas de manejo integrado de moscas frugívoras, se hace necesario conocer aspectos básicos de su biología y ecología (Aluja et al., 2003). Birke et al. (2015) mencionan como aspecto fundamental para prevenir la introducción de plagas cuarentenarias, conocer el uso de hospederos por parte de estas moscas; adicionalmente, el uso de hospederos por tefrítidos y su competencia inter e intra específica es un tema que se ha estudiado a nivel de ensayos en campo y laboratorio (Duyck et al., 2004; Sivinski 
et al., 2004; Aluja y Díaz-Fleischer, 2006; Segura et al., 2006; Devescovi et al., 2015; Lopes et al., 2015; Liendo et al., 2016). Cuando los tefrítidos invaden nuevas áreas, se puede presentar competencia jerárquica o coexistencia estable con especies preestablecidas. La competencia jerárquica ocurre cuando una especie domina o excluye a la otra y la coexistencia estable consiste en la diferenciación o fragmentación de nichos por la ausencia de la especie más competitiva (Duyck et al., 2004). Estos autores observaron que en la mayoría de los casos se presentó una competencia jerárquica, pero sin una exclusión completa de la otra especie; sin embargo, algunos factores como la calidad de frutos, condiciones ambientales, localización de nicho, especificidad de las moscas, feromonas de marcaje, entre otros, se han puesto en discusión para poder determinar la variación de los comportamientos de competencia entre tefrítidos por un mismo recurso (Segura et al., 2006; Silva et al., 2012).

Son pocos los trabajos que buscaron estudiar en campo la competencia de especies de moscas por hospederos y más escasos lo referente al uso de un mismo fruto por moscas diferentes. Estos trabajos requieren el muestreo y el análisis de los frutos individuales. Tan solo González y Carrejo (1993), Birke y Aluja (2011), Devescovi et al. (2015), individualizaron los frutos, en los cuales se observó que la coexistencia en un mismo fruto es poco frecuente, y cuando se encontraron infestando la misma especie de planta hospedera, en la mayoría de los casos se presenta la predominancia de una especie sobre la otra. Aluja y Díaz-Fleischer (2006) realizaron un ensayo con feromonas de marcaje, donde encontraron que hembras de Anastrepha tienden a buscar y seleccionar frutos que se encuentran sin marcar, de ahí que las feromonas de marcaje en este género son importantes a la hora de evitar la oviposición de frutos ya infestados por otras especies.
Aunque se han desarrollado programas de cría de especies de parasitoides nativos de las familias Braconidae y Figitidae, para el control de especies de tefrítidos (Aluja et al., 2009; Gonçalves et al., 2016), en Colombia la mayoría de trabajos realizados se han limitado a conocer la diversidad tanto de moscas frugívoras como de sus parasitoides (Yepes y Vélez, 1989; Portilla et al., 1994; Carrejo y González, 1999; Núñez et al., 2004b; Castañeda et al., 2010). Tan solo Sarmiento et al. (2012) y Ruiz-Hurtado et al. (2013), han reportado datos de ecología de estas asociaciones tróficas en zonas cafeteras del país; por lo tanto, es necesario conocer los aspectos ecológicos y el uso de hospederos de las moscas frugívoras y sus parasitoides, como base para futuros programas de control biológico (Aluja et al., 2009; Garcia y Ricalde, 2013).

Elobjetivo de estetrabajo fue reconocer eidentificar la diversidad de especies de moscas de las frutas y sus parasitoides asociados, describiendo las relaciones tróficas entre plantas hospederas, moscas frugívoras y sus parasitoides, en un parche de bosque seco tropical en el Centro Universitario Regional del Norte (Armero Guayabal, Tolima), como un aporte al conocimiento de este grupo de insectos y ofrecer información ecológica básica que soporte futuras decisiones en programas de manejo de estas plagas.

\section{MATERIALES Y MÉTODOS}

El estudio se realizó en el Centro Universitario Regional del Norte (CURDN), Universidad del Tolima, localizado en el municipio de Armero Guayabal, al norte del departamento del Tolima con coordenadas $5^{\circ} 00^{\prime} 05^{\prime \prime} \mathrm{LN}$ y $74^{\circ} 54^{\prime} 27^{\prime \prime} \mathrm{LO}$. El CURDN tiene un área aproximada de 700ha, que comprende zonas de cultivos agrícolas, forestales, pecuarias y de bosque nativo. La temperatura promedio anual es de $27^{\circ} \mathrm{C}$, la precipitación media anual es de $1.738 \mathrm{~mm}$ y la humedad relativa anual 
es de $71 \%$. La altura oscila entre 275 a $550 \mathrm{msnm}$ ubicándose en la zona de vida de bosque seco tropical Bs-T (Universidad del Tolima, 2012).

Entre abril a octubre de 2013 se realizó el muestreo de frutos de diferentes especies de plantas, tanto de frutos silvestres como de frutos de huertos no comerciales. Los frutos se colectaron directamente de la planta y/o del suelo según su disponibilidad. Las muestras obtenidas se acondicionaron en recipientes plásticos aireados con una pequeña cantidad de vermiculita y fueron transportadas hasta el Laboratorio del Museo de Entomología de la Universidad del Tolima MENT-UT, Ibagué, Tolima.

El procesamiento de las muestras para la separación de larvas y pupas se realizó adaptando la metodología desarrollada por Uchôa-Fernandes y Zucchi (1999), la cual permite realizar una asociación precisa entre moscas frugívoras y sus parasitoides. Para esto, cuando llegaron las muestras al laboratorio, se contaron y pesaron los frutos de cada muestra. Cada fruto fue dispuesto individualmente sobre una superficie de tela dentro de recipientes plásticos o de icopor que variaron sus dimensiones según el tamaño de la fruta. A los recipientes se les colocaba una lámina de 15 a $20 \mathrm{~mm}$ de agua para la colecta de las larvas maduras. El agua de los recipientes era escurrida en un cernidor cada 12 horas para la colecta, conteo y separación de las larvas. Las larvas/ pupas se separaban según la familia (Tephritidae y Lonchaeidae) utilizando características de los espiráculos posteriores y el tamaño, forma y color del cuerpo (Uchôa-Fernandes y Zucchi 1999; Nicácio y Uchôa, 2011) y se colocaban en frascos con vermiculita humedecida para la emergencia de adultos de moscas y/o parasitoides. Al cabo de 30 días, los frutos se disectaron para registrar y separar las larvas o pupas que todavía permanecían en los frutos. Las condiciones ambientales (temperatura y humedad) de las muestras proce- sadas no fueron controladas, variando según las condiciones del laboratorio y solo se controló el fotoperiodo (12:12 h.).

La identificación de las moscas Tephritidae se realizó por medio de caracteres morfológicos como el diseño torácico, el diseño alar y la genitalia femenina, usando claves específicas y textos taxonómicos (Korytkowski, 2008; Norrbom et al., 2012). Los Lonchaeidae se identificaron según el diseño de la cabeza, el diseño torácico y la genitalia del macho, usando para los géneros las claves de McAlpine (1987); MacGowan y Okamoto (2013). Para las especies de Lonchaea Fallén se basó en el trabajo de Korytkowski y 0jeda (1971) y para las especies de Neosilba McAlpine se utilizaron las claves de McAlpine y Steyskal (1982); Galeano-Olaya y Canal (2012). Los parasitoides obtenidos se identificaron analizando la morfología bucal (mandíbulas), nerviación alar, las tibias y el propodeum. La identificación se realizó utilizando claves específicas (Canal y Zucchi, 2000; Guimarães et al., 2000; Wharton y Yoder, 2014). Los especímenes de moscas y parasitoides se depositaron en la colección del MENT-UT.

Se calculó el porcentaje de daño $[\% \mathrm{D}=$ (número de frutos con larvas / total de frutos analizados) $* 100]$, el índice de infestación [I = (número de larvas obtenidas de una muestra de fruta / peso de la muestra o número de frutas de la muestra)], el porcentaje de viabilidad de larvas de tercer instar $[\% \mathrm{VL}=$ (número de moscas emergidas / (número total de larvas de tercer instar - número de parasitoides emergidos) $* 100]$, el porcentaje de parasitismo [\% $\mathrm{P}=$ (número de parasitoides emergidos / número de larvas de moscas frugívoras) * 100] y frecuencia de ocurrencia de parasitoides [\% $\%$ = (número de individuos de una determinada especie de parasitoide / número total de parasitoides) x 100] (Uchôa-Fernandes et al., 2003; Núñez et al., 2004a). 
Las plantas hospederas se determinaron y confirmaron con ayuda del personal del Herbario Toli y el Laboratorio de Dendrología "Fernando Aly Huertas Gómez" de la Universidad del Tolima. Los vouchers de plantas hospederas se depositaron en la colección científica del Hernbario Toli.

\section{RESULTADOS Y DISCUSIÓN}

Se coleccionaron 165 muestras de frutos correspondientes a 35 especies de 21 familias de plantas, procesando 3.174 frutos con un peso de $113,014 \mathrm{~kg}$ (Tabla 1). Del total de muestras de frutos colectadas, el 47\% (78 muestras) pertenecientes a 14 especies de plantas y 10 familias, presentaron ataque por moscas frugívoras, 43 de ellas fueron colectadas del árbol y 35 del suelo (Tabla 2). Se colectaron 2.940 larvas de moscas frugívoras Tephritoidea, de las cuales 2.458 pertenecían a Tephritidae y 482 a Lonchaeidae. Emergieron 2.285 adultos, pertenecientes a seis especies de Tephritidae $(82,49 \%$ de los adultos obtenidos) y ocho especies de Lonchaeidae $(17,51 \%$ de los adultos) (Tabla 2$)$.

Tephritidae. Los tefrítidos se asociaron a siete plantas hospederas de cinco familias. Se registraron cinco especies de Anastrepha (A. montei Lima, A. obliqua (Macquart), A. pickeli Lima, A serpentina (Wiedemann) y A. striata Schiner) y Toxotrypana curvicauda Gerstaecker. La especie más abundante fue $A$. striata (1.246 individuos) asociada a Psidium guajava, seguida de A. obliqua (419 individuos) colectada de frutos de Mangifera indica, Plinia cauliflora y Psidium guajava, en este último hospedero solo se obtuvo un individuo. A. montei y A. pickeli se registraron en Manihot esculenta y A. serpentina se asoció a frutos de Mangifera indica, Manilkara zapota y Pouteria caimito. Entretanto T. curvicauda fue la única especie de tefrítido asociado a $\mathrm{Ca}$ rica papaya $\mathrm{L}$.
En referencia a los porcentajes de daño causados, considerados como el número de frutos afectados en relación al total de frutos, los mayores valores por moscas Tephritidae se registraron en Psidium guajava, Manihot esculenta y Mangifera indica con 88,31, 65,79 y 56,72\%, respectivamente. Los más bajos sucedieron en Pouteria caimito $(0,85 \%)$ y Manilkara zapota (3,39\%) (Tabla 1). La viabilidad de larvas de tercer estadio para Tephritidae vario entre 53,33 y $100 \%$, con un viabilidad media de 74,87\% (Tabla 2). Los índices de infestación por tefrítidos en muestras obtenidas del árbol variaron en un rango de 0 a 9,27 larvas/fruto y de 0 a 463,37 larvas/kg frutos. En las muestras del suelo, los índices de infestación variaron entre 0 a 10,32 larvas/fruto y de 0 a 156,88 larvas/kg fruto. Por otro lado, la capacidad de albergar un mayor número de larvas por fruto se registró en Psidium guajava, Mangifera indica y Pouteria caimito con rangos entre 1-49, 1-40 y 30 larvas/fruto (Tabla 3).

Lonchaeidae. Los lonquéidos fueron más generalistas, encontrados en 12 plantas hospederas de nueve familias. Se registraron seis especies de Neosilba N. batesi (Curran), N. bifida Strikis y Prado, N. certa (Walker), N. glaberrima (Wiedemann), N. pendula (Bezzi) y N. zadolicha McAlpine y Steyskal) y dos especies de Lonchaea (L. longicornis Williston y L. striatifrons Malloch). Tan solo en dos plantas hospederas (Maclura tinctoria y Manilkara zapota) se registraron larvas de lonquéidos, sin que se formaran adultos. $N$. batesi fue la especie más abundante con 146 individuos, registrados en cuatro hospederos, seguida de $N$. glaberrima $(79$ individuos) que es la más polífaga encontrada en frutos de cinco especies de plantas. En cuatro plantas hospederas (Annona cherimola, Carica papaya, Mangifera indica y Rollinia mucosa) se registraron más de una especie de moscas, el resto solo albergo una especie de lonquéideo (Tabla 2). 
Tabla 1. Hospederos y daños de moscas frugívoras (Diptera: Tephritoidea) en el CURDN, Armero Guayabal, Tolima.

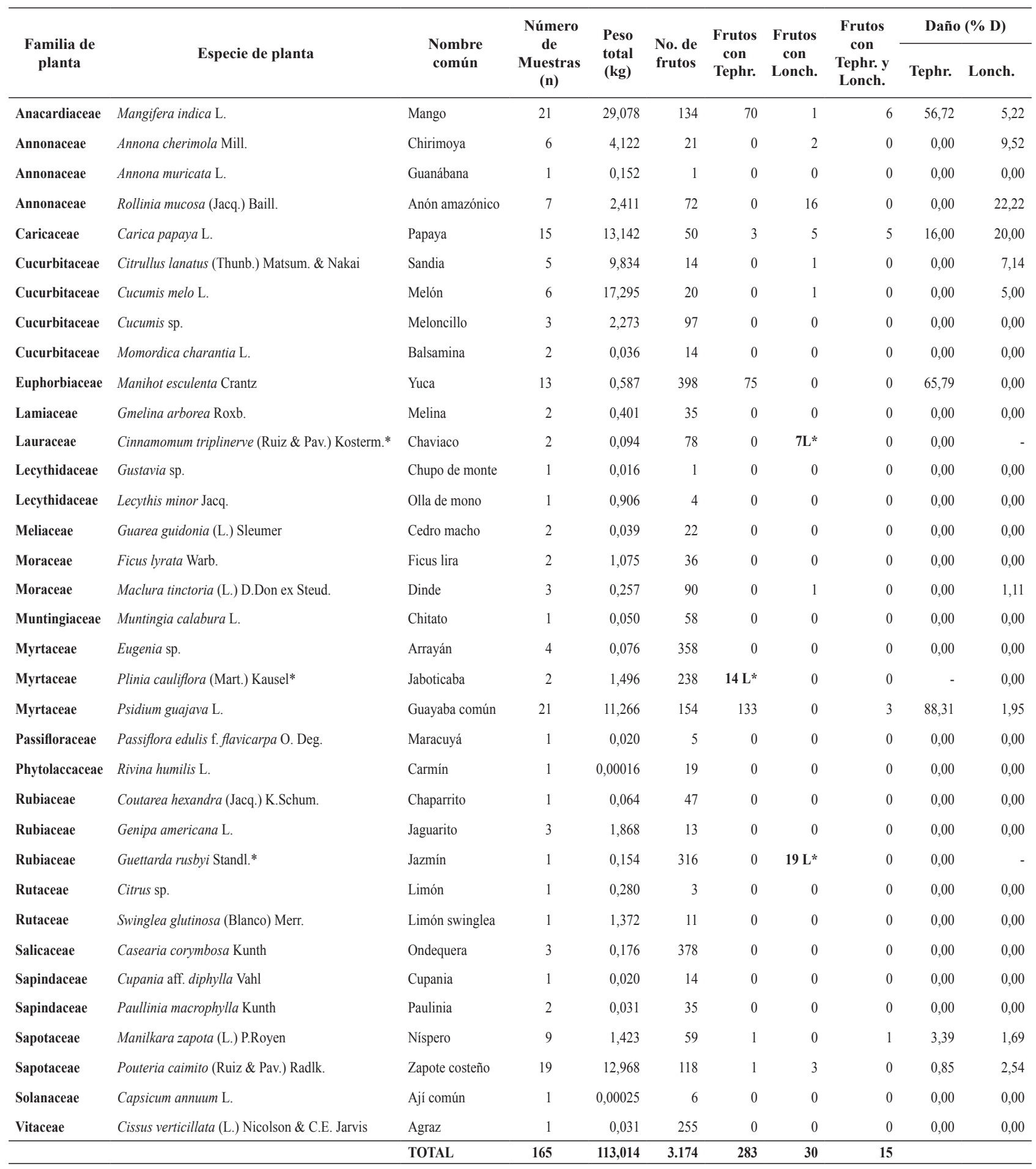

* Especies de plantas hospederas donde no se individualizaron los frutos colectados, el dato corresponde al número de larvas obtenidas. 


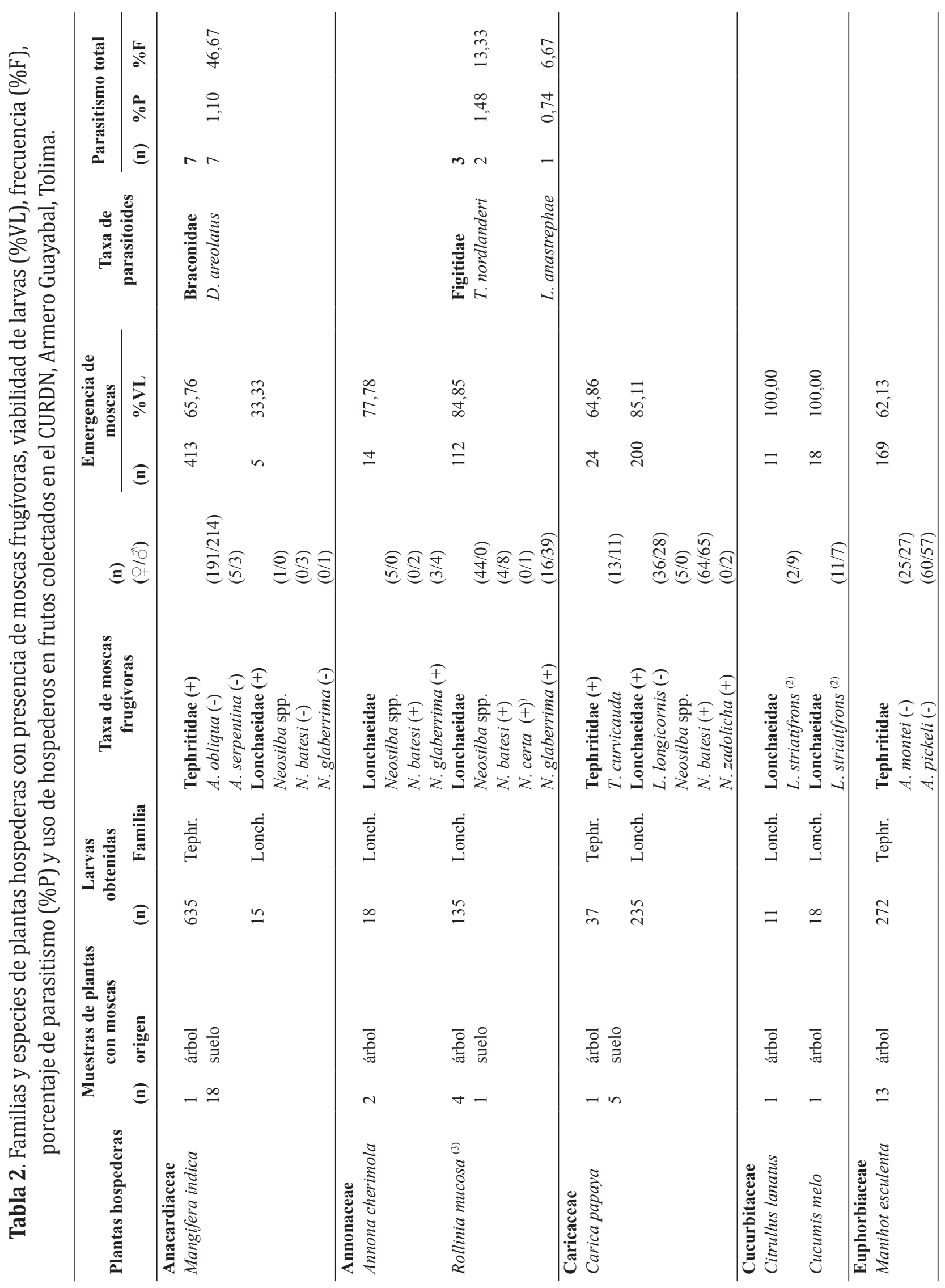




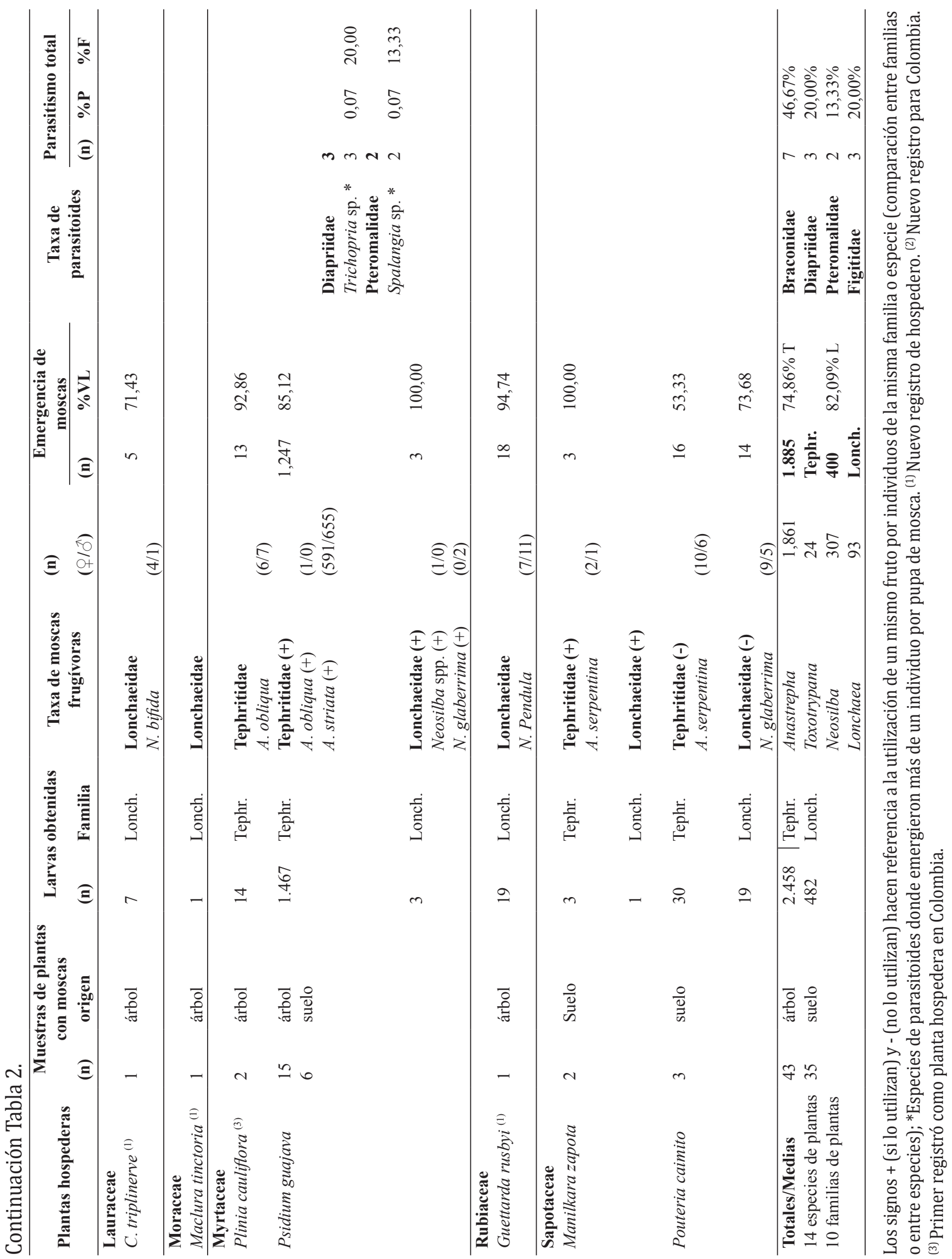


Los más altos porcentajes de daño por moscas Lonchaeidae ocurrieron en Rollinia mucosa $(22,22 \%)$ y Carica papaya (20,00\%), y los más bajos sucedieron en Maclura tinctoria (1,11\%), Manilkara zapota $(1,69)$ y Psidium guajava $(1,95 \%)$ (Tabla 1). La viabilidad de larvas de tercer estadio para Lonchaeidae vario entre 33,33\% y $100 \%$, con un viabilidad media de $82,09 \%$ (Tabla 2). Por otro lado, los índices de infestación en muestras colectadas del árbol variaron en un rango de 0 a 2,06 larvas/fruto y de 0 a 123,38 larvas/kg frutos. En las muestras del suelo, los índices de infestación variaron entre 0 a 8,03 larvas/ fruto y de 0 a 68,63 larvas/kg fruto. Los mayores rangos de infestación por fruto se registraron en Carica papaya y Rollinia mucosa entre 1-69 y 1-32 larvas/fruto respectivamente (Tabla 3).
Uso de hospederos por moscas frugívoras. De las 14 plantas hospederas que registraron moscas frugívoras, en cinco se registraron las dos familias de moscas y en su mayoría se presentaron frutos infestados por ambas familias, a excepción de Pouteria caimito donde se registraron las dos familias pero en frutos distintos. Las plantas hospederas que mayor número de especies de moscas frugívoras presentaron fueron Mangifera indica y Carica papaya, con cuatro especies. Psidium guajava y Rollinia mucosa fueron infestadas por tres especies de moscas frugívoras, mientras que Annona cherimola, Manihot esculenta y Pouteria caimito fueron atacadas por dos especies cada una; el resto de plantas (6) fueron hospederas de solamente una especie de Lonchaeidae (4) o de Tephritidae (2), sin embargo, en siete plantas hospederas se registraron solo lonquéidos y dos plantas albergaron solo tefrítidos (Tabla 2).

Tabla 3. Índices de infestación y rangos del índice de infestación por moscas frugívoras (Diptera: Tephritoidea) en frutos colectados del árbol y del suelo en el CURDN. Armero Guayabal, Tolima.

\begin{tabular}{|c|c|c|c|c|c|c|c|c|}
\hline \multirow{3}{*}{$\begin{array}{c}\text { Plantas } \\
\text { hospederas }\end{array}$} & \multirow{2}{*}{\multicolumn{2}{|c|}{$\begin{array}{l}\text { Número total de } \\
\text { larvas }\end{array}$}} & \multicolumn{4}{|c|}{ Índices de infestación para todos los frutos } & \multicolumn{2}{|c|}{$\begin{array}{l}\text { Rango del índice de infestación } \\
\text { para frutos con moscas }\end{array}$} \\
\hline & & & \multicolumn{2}{|c|}{ Tephritidae } & \multicolumn{2}{|c|}{ Lonchaeidae } & \multirow{2}{*}{$\begin{array}{l}\text { Tephritidae } \\
\text { larvas / fruto }\end{array}$} & \multirow{2}{*}{$\begin{array}{c}\text { Lonchaeidae } \\
\text { larvas / fruto }\end{array}$} \\
\hline & Tephr. & Lonch. & $\begin{array}{c}\text { larvas / } \\
\text { fruto }\end{array}$ & $\begin{array}{l}\text { larvas / } \mathrm{kg} \\
\quad \text { frutos }\end{array}$ & $\begin{array}{l}\text { larvas / } \\
\text { fruto }\end{array}$ & $\begin{array}{l}\text { larvas / } \mathrm{kg} \\
\quad \text { frutos }\end{array}$ & & \\
\hline \multicolumn{9}{|l|}{ ARBOL } \\
\hline Annona cherimola & 0 & 18 & 0 & 0 & 0,95 & 4,71 & 0 & $4-14$ \\
\hline Carica papaya & 0 & 2 & 0 & 0 & 0,10 & 0,21 & 0 & 2 \\
\hline C. triplinerve* & 0 & 7 & 0 & 0 & 0,09 & 74,47 & 0 & - \\
\hline Citrullus lanatus & 0 & 11 & 0 & 0 & 0,79 & 1,12 & 0 & 11 \\
\hline Cucumis melo & 0 & 18 & 0 & 0 & 0,90 & 1,04 & 0 & 18 \\
\hline Guettarda rusbyi* & 0 & 19 & 0 & 0 & 0,06 & 123,38 & 0 & - \\
\hline Maclura tinctoria & 0 & 1 & 0 & 0 & 0,01 & 4,27 & 0 & 1 \\
\hline Mangifera indica & 40 & 0 & 6,67 & 31,40 & 0 & 0 & $2-25$ & 0 \\
\hline Manihot esculenta & 272 & 0 & 0,68 & 463,37 & 0 & 0 & 1 & 0 \\
\hline Plinia cauliflora* & 14 & 0 & 0,06 & 9,36 & 0 & 0 & - & 0 \\
\hline Psidium guajava & 1.085 & 3 & 9,27 & 122,86 & 0,03 & 0,34 & $1-49$ & 1 \\
\hline Rollinia mucosa & 0 & 130 & 0 & 0 & 2,06 & 58,01 & 0 & $1-32$ \\
\hline \multicolumn{9}{|l|}{ SUELO } \\
\hline Carica papaya & 37 & 233 & 1,28 & 10,90 & 8,03 & 68,63 & $1-9$ & $1-69$ \\
\hline Mangifera indica & 595 & 15 & 4,65 & 21,40 & 0,12 & 0,54 & $1-40$ & $1-5$ \\
\hline Manilkara zapota & 3 & 1 & 0,06 & 2,84 & 0,02 & 0,95 & $1-2$ & 1 \\
\hline Pouteria caimito & 30 & 19 & 0,31 & 3,43 & 0,20 & 2,17 & 30 & $1-13$ \\
\hline Psidium guajava & 382 & 0 & 10,32 & 156,88 & 0 & 0 & $1-38$ & 0 \\
\hline Rollinia mucosa & 0 & 5 & 0 & 0 & 0,56 & 29,41 & 0 & 5 \\
\hline
\end{tabular}

* Especies de plantas hospederas donde no se individualizaron los frutos colectados. 
De las seis especies de plantas hospederas donde se registraron especies de Anastrepha, tan solo en tres especies (Mangifera indica, Manihot esculenta y Psidium guajava) se observó más de una especie de Anastrepha. En Mangifera indica se registraron A. obliqua y $A$. serpentina, en donde tan solo se registró un fruto atacado exclusivamente por $A$. serpentina. Manihot esculenta albergo dos especies del grupo "Spatulata", pero no cohabitaron un mismo fruto, y en Psidium guajava se encontró un fruto donde emergieron las especies $A$. striata y A. obliqua, pero de esta última tan solo emergió un individuo. En las cuatro especies de plantas hospederas donde se registraron más de una especie de lonquéido, estas compartieron el mismo recurso o nicho, en el caso de Rollinia mucosa se encontró en un mismo fruto tres especies de Neosilba cohabitando (Tabla 2).

Parasitoides. Se coleccionaron cinco especies de parasitoides, los cuales emergieron de larvas infestando tres especies de plantas hospederas: Doryctobracon areolatus (Szépligeti) (Braconidae), Trybliographa nordlanderi (Wharton), Lopheucoila anastrephae (Rohwer) (Figitidae), Trichopria sp. (Diapriidae) y Spalangia sp. (Pteromalidae). Se obtuvieron 15 individuos de parasitoides, siendo D. areolatus $(46,67 \%)$ la especie más abundante asociada a A. obliqua en frutos de Mangifera indica; Trichopria sp. (20,00\%) y Spalangia sp. $(13,33 \%)$ se encontraron en frutos de Psidium guajava infestados por A. striata. Las dos especies de Figitidae, T. nordlanderi (13,33\%) y L. anastrephae $(6,67 \%)$, se encontraron asociadas a moscas Neosilba spp. en frutos de Rollinia mucosa. En los casos donde una planta hospedera albergo dos especies de parasitoides, no se encontró más de una especie de parasitoide por fruto; sin embrago, de las especies de Trichopria sp. y Spalangia sp. emergieron más de un individuo por pupa de mosca. Los porcentajes de parasitismo variaron entre 0,07 a 1,44\% (Tabla 2).
En el Tolima hasta el momento se habían realizado levantamiento de moscas de las frutas en 10 municipios (Castañeda et al., 2010) y trabajos relacionados con parasitoides solo en los municipios de Rovira y Líbano (Ruiz-Hurtado et al., 2013); por tal razón, todas las moscas frugívoras y sus parasitoides reportados en este trabajo, son el primer registro para el municipio de Armero Guayabal (Tolima).

Lonchaea striatifrons (Diptera: Lonchaeidae) se registran por primera vez para Colombia. L. striatifrons había sido registrada previamente para Perú, Suroeste de los Estados Unidos, México y Hawái (Korytkowski y 0jeda 1971). Cinnamomum triplinerve se registra como nueva planta hospedera de Neosilba pendula; Rollinia mucosa por primera vez se reporta como hospedero de Neosilba en Colombia y Plinia cauliflora hospedando Anastrepha obliqua en Colombia (Tabla 2).

Porcentajes de daño e índices de infestación. Los porcentajes de daño se lograron establecer para los frutos que se individualizaron, donde los porcentajes más altos fueron para los tefrítidos. Núñez et al. (2004a) registraron por dos años el porcentaje de daño en frutos de Coffea arabica L. y Psidium guajava. Este último alcanzó en los dos años un promedio de $98,03 \%$ de daño en frutos. Portilla et al. (1994) y Sarmiento et al. (2012) presentaron porcentajes de daño únicamente para Coffea arabica y Spondias mombin L. respectivamente. Para lonquéidos, los trabajos realizados en pasifloras han mostrado daños hasta del 100\% en frutos y botones florales (Quintero et al., 2012; Wyckhuys et al., 2012; Santamaría et al., 2014).

Psidium guajava fue la especie con mayores índices de infestación para Tephritidae, tanto de muestras del suelo como del árbol, estos valores son similares a los obtenidos por Núñez et al. (2004a). Manihot esculenta, presentó el mayor número de larvas/kg frutos, pero nunca se encontró más de 
una larva por fruto debido principalmente a su tamaño. En realidad ya es conocido que los mayores índices de infestación ocurren en frutos pequeños (Uchôa-Fernandes et al., 2003). Sin embargo, Núñez et al. (2004a) registraron el caso contrario en frutos de guayaba y café, explicando que la infestación por moscas varía dependiendo de las características del fruto, la especie de tefrítido y a la ausencia de hospederos alternantes. En Colombia no existe información sobre índices de infestación de lonquéidos, tan solo Santamaría et al. (2014) mostraron el promedio de larvas de Dasiops spp. en distintos órganos de pasifloras con un máximo de 2 larvas/órgano. Portilla et al. (1994) presentaron porcentajes de infestación e índices de infestación solo para tefrítidos en Café, mientras que Ruiz-Hurtado et al. (2013) registraron índices de infestación sin especificarlos para cada familia de moscas frugívoras.

Los lonquéidos fueron más polífagos que los tefrítidos, pero estos últimos fueron los que causaron mayor porcentaje de daño y alcanzaron los índices de infestación más altos en la mayoría de plantas atacadas por moscas frugívoras. Estos resultados concuerdan con lo encontrado por Ruiz-Hurtado et al. (2013) en dos zonas cafeteras del Tolima, zonas geográficas muy distintas a las del CURDN. También en Brasil, Bomfin et al. (2014) encontraron que larvas de Neosilba lograron infestar un mayor número de hospederos que Anastrepha, por lo que este grupo de insectos han sido considerados de importancia económica por la alta polifagia e índices de infestación que han presentado en este país tanto en plantas nativas como exóticas (Uchôa, 2012). En Colombia especies del genero Dasiops han sido las de mayor importancia de estudio por los daños que han causado en cultivos de pasifloras (Delgado et al., 2010b; Quintero et al., 2012; Wyckhuys et al., 2012; Santamaría et al., 2014), sin embargo, individuos del genero Neosilba han sido reportados asociados con la pudrición basal en pitahaya amarilla
(Delgado et al., 2010a) y así mismo especies de Lonchaea y Neosilba en daños a botones florales en esta misma especie de planta (Medina y Kondo, 2012).

La situación contrastante entre la polifagia de lonquéidos y la gran capacidad de infestación y de daño de los tefrítidos, puede estar fundamentado en la hipótesis que los parámetros demográficos naturales (tasa de reproducción, tasa intrínseca de crecimiento, tiempo medio generacional, entre otros) de tefrítidos pueden ser mayores a los que presentan los lonquéidos, sin embargo, son pocos los trabajos que abordan aspectos de biología y ecología básica de Lonchaeidae. Apenas Nicácio y Uchôa (2011) observaron el tiempo de pupación de tefrítidos y lonquéidos colectados en muestras de frutas; estos autores observaron que especies de Neosilba tienen un periodo más corto de pupación y las hembras pueden presentar madurez sexual antes que los machos; además, consideran que los lonquéidos son uno de los grupos de Tephritoidea más primitivos, por lo que podrían presentar un sistema más antiguo de apareamiento.

Uso de hospederos por moscas frugívoras. En relación con el uso compartido de hospederos, se observó que tefrítidos (especies de Anastrepha) y lonquéidos (especies de Neosilba) infestaron un mismo recurso (en los casos de Mangifera indica, Carica papaya, Psidium guajaba y Manilkara zapota), además se corroboró que ambas familias de moscas permitieron el uso compartido de frutos sin que se afecte el desarrollo de las mismas. Resultados similares fueron registrados por RuizHurtado et al. (2013) en Colombia y por Nicácio y Uchôa (2011) y Bomfin et al. (2014) en Brasil, quienes encontraron especies de Anastrepha y de Neosilba compartiendo plantas hospederas. Díaz-Fleischer et al. (2000) menciona que los estados inmaduros de lonquéidos se desarrollan en recursos como madera en descomposición, estiércol, insectos, hongos y plantas, contrario a las 
especies de Tephritidae que se han especializado a un cierto grupo de recursos principalmente frutos de plantas y que además, ésta especialización ha promovido la evolución de este grupo siendo el más diverso de la Superfamilia.

Por otra parte, se observó que lonquéidos compartieron hospederos entre sí, contrario a lo observado en tefrítidos donde muy rara vez compartieron plantas hospederas con especies de la misma familia. Esta situación ha sido confirmada anteriormente por Aluja et al. (2003) quienes consideran que especies de Anastrepha tienden a no compartir sus hospederos, y en casos particulares donde esto sucede puede ser considerada como una excepción. En la mayoría de los casos donde se encuentra varias especies polífagas de Anastrepha, las moscas más abundantes son las que se encuentran asociadas comúnmente a estos hospederos, como en el caso de A. obliqua a Mangifera indica o A. striata a Psidium guajava y A. serpentina a Pouteria caimito.

En la zona de estudio, bosque seco tropical, no existen variaciones climáticas extremas como ocurre en el subtrópico, facilitando la presencia de al menos pocos frutos hospederos la mayor parte del año, lo cual puede influir en que las especies de Anastrepha estén asociadas solo a sus hospederos preferidos. En otros casos, se han encontrado relaciones de co-ocurrencia con especies exóticas de tefrítidos. En Argentina Segura et al. (2006) lograron observar que Ceratitis capitata y Anastrepha obliqua compartieron plantas hospederas en distintas zonas, presentándose una abundancia de la una o de la otra en distintos hospederos. Lopes et al. (2015) analizaron los patrones de co-ocurrencia por par de especies de tefrítidos en tres plantas hospederas, encontrando que 11 pares de especies eran segregadas y en tan solo siete fueron agregadas, donde Ceratitis capitata fue la especie que más se involucró en las asociaciones.
Las especies de Anastrepha que compartieron hospedero, no se encontraron coinfestando un mismo fruto. Tan solo en Psidium guajava las dos especies de Anastrepha emergieron del mismo fruto, pero solo se encontró un espécimen de $A$. obliqua en este hospedero, lo cual indica apenas un uso ocasional. En México, Birke y Aluja (2011) registraron tan solo en el $4 \%$ de los frutos individualizados, la emergencia de dos especies de Anastrepha (A. striata y A. obliqua) en un mismo fruto (P.guajava), lo cual puede indicar un reconocimiento de frutas previamente infestadas por otras especies. Estos mismos autores sugieren que esta situación de coinfestación se puede presentar por la escasez de hospederos principales y quizás por confusión de las especies de moscas a características externas parecidas a la de sus hospederos principales. Así mismo, Devescovi et al. (2015) observaron que las especies Ceratitis capitata y Anastrepha fraterculus, tienden a no preferir para su oviposición fruta que ya se encuentra infestada por la otra especie.

Aunque se han reportado infestaciones simultaneas de especies de Anastrepha en un mismo fruto (González y Carrejo, 1993; Birke y Aluja, 2011), se ha demostrado anteriormente que en la mayoría de especies el uso de feromonas de marcaje de hospedero juega un papel fundamental para prevenir el uso compartido de un mismo fruto por distintas especies (Aluja y Díaz-Fleischer, 2006 ; Silva et al., 2012), e incluso llegar a tener implicaciones en las relaciones intra-especificas, evitando varias posturas de una especie en el mismo fruto (González y Carrejo, 1993). Aluja y Díaz-Fleischer (2006) observaron que especies de Anastrepha reconocen las feromonas de marcaje de otras especies, y que así mismo tienden a buscar y preferir frutos que no se encuentran marcados con feromonas. En la revisión realizada por Duyck et al. (2004), observaron que en la mayoría de los casos donde se presentó competencia por espacio entre una especie invasiva con otra ya preestablecida, hubo una dominación del nicho por parte de una especie, sin excluir 
totalmente a la otra. En estos casos, la exclusión puede estar referida al uso del mismo fruto. En lonquéidos no existe información sobre feromonas de marcaje, además se observó que tienden a ser más polífagas y compartir el mismo recurso (fruto) entre ellas y con especies de otras familias.

Birke y Aluja (2011), consideran que es importante estudiar más a fondo la capacidad que presentan especies polífagas de compartir recursos. Esto permitiría conocer las interacciones de especies simpátricas de moscas de la fruta, fundamental para el manejo de plagas de importancia económica.

Uso de hospederos por parasitoides. Los parasitoides de las familias Braconidae, Diapriidae y Pteromalidae se asociaron a larvas de Tephritidae y las especies de la familia Figitidae se encontraron atacando exclusivamente larvas de Lonchaeidae. Trabajos anteriores (Uchôa-Fernandes et al., 2003; Nicácio et al., 2011) han observado parasitoides de la familia Figitidae emergiendo principalmente de larvas de lonquéidos y parasitoides Braconidae atacando frecuentemente especies de Tephritidae; aunque se han desarrollado trabajos de cría de parasitoides figitidos para el control de especies de moscas Tephritidae (Aluja et al., 2009; Gonçalves et al., 2016). En la revisión hecha en Brasil por Garcia y Ricalde (2013), se observa que especies de braconidos han sido registrados atacando principalmente especies de Anastrepha. Ruiz-Hurtado et al. (2013) consideran que parasitoides figitidos pueden tener una preferencia natural por larvas de Lonchaeidae en la zona del neotrópico. Cuando se encuentran moscas frugívoras de ambas familias en una planta hospedera, la metodología de colección comúnmente utilizada puede causar errores de asociación entre parasitoides y hospederos, lo cual puede dificultar acciones de control biológico aplicado (Uchôa y Zucchi, 1999; Uchôa-Fernandes et al., 2003).
Doryctobracon areolatus fue el parasitoide más abundante. En Colombia las especies de parasitoides se distribuyen en rangos altitudinales diferentes, siendo Doryctobracon crawfordi y Utetes anastrephae las especies que predominan en zonas altas, mientras D. areolatus es un parasitoide abundante en áreas bajas, como es el bosque seco tropical donde se realizó el presente estudio (Núñez et al., 2004b; Sarmiento et al., 2012; Ruiz-Hurtado et al., 2013). Sarmiento et al. (2012) observaron infestación por tres especies de Anastrepha y por cinco especies de parasitoides, donde Anastrepha obliqua fue la especie más abundante atacando frutos de Spondias. De este hospedero se registraron nueve individuos de $D$. areolatus pero no se logró hacer una asociación clara, debido a que se registró más de una especie de mosca en las muestras de frutas. Núñez et al. (2004b) en cafetales de Santander hallaron que la especie predominante en esa zona de estudio fue D. crawfordi atacando larvas de A. striata y $A$. fraterculus en frutos de guayaba y café. En Brasil, Garcia y Ricalde (2013) mencionan a D. Areolatus, un parasitoide ampliamente distribuido en ese país y con otras especies de braconidos (Opius bellus Gahan y Utetes anastrephae Viereck), como los más promisorio para ser utilizado en programas de control biológico en contra de especies de Anastrepha.

En frutos de guayaba emergieron dos especies de parasitoides (Trichopria sp. y Spalangia sp.) de $A$. striata. Trichopria sp. es mencionado por Ovruski et al. (2000) como endoparasitoides idiobiontes de pupas, este mismo autor explica que estos parasitoides atacan a sus hospederos después de empupar en el suelo. En Brasil, Uchôa-Fernandes etal. (2003) registraron dos especies de Spalangia parasitando pupas de Tephritidae y Lonchaeidae, y Nicácio et al. (2011) registraron Spalangia en larvas de Tephritidae; Wharton y Yoder (2014) refieren este género como un parasitoide pupal polífago, el cual se ha registrado en tefrítidos. 
Cabe mencionar, la poca probabilidad que utilizando la metodología del sustrato de agua para colectar y separar las larvas que salen de los frutos a empupar, se obtengan parasitoides de tipo pupal como sucede con las dos especies de parasitoides encontradas en este trabajo, teniendo solo dos alternativas: 1) contaminación de los medios de empupación/emergencia en el laboratorio, y 2) la posibilidad que estas especies hayan parasitado en estado larval a los tefrítidos como lo mencionan Nicácio et al. (2011). Cabe resaltar que donde fueron mantenidas las pupas para la emergencia de adultos se mantienen colonias de $A$. fraterculus y A. obliqua que nunca han sido atacadas por parasitoides de pupas, por lo tanto, éstas especies atacaron las larvas en el campo.

Se asociaron a larvas de Neosilba dos especies de parasitoides figitidos en frutos de Rollinia mucosa. Trybliographa nordlanderi posiblemente emergió de pupas de la especie $N$. glaberrima; la asociación precisa se dificultó debido a que del mismo fruto emergió más de una especie de Neosilba. Ruiz-Hurtado et al. (2013) registraron por primera vez T. nordlanderi para Colombia, asociado a larvas de $A$. fraterculus, A. striata y Neosilba sp. en frutos de Coffea arabica, Psidium guajava y Capsicum frutescens, sin embargo, estos autores no individualizaron frutos. Lopheucoila anastrephae se asoció a $N$. glaberrima en frutos de Rollinia mucosa, Ruiz-Hurtado et al. (2013) registraron a $L$. anastrephae en pupas de lonquéidos que infestaron Coffea arabica. Las dos especies de figitidos son frecuentes en la región neotropical, atacando larvas del género Neosilba las cuales han sido consideradas de importancia económica en países como Brasil (Uchôa, 2012). Esta situación perfila a parasitoides Figitidae como una alternativa para ser utilizados en programas de manejo integrado de moscas Lonchaeidae, siendo necesario desarrollar trabajos sobre biología básica y comportamiento tanto de lonquéidos como de sus parasitoides (Uchôa, 2012).
Los bajos niveles de parasitismo se observaron debido a la poca abundancia de las especies de parasitoides, registrando tan solo 15 especímenes en total. Ruiz-Hurtado et al. (2013) mencionan que la abundancia de larvas de moscas frugívoras, pueden influir positivamente en la abundancia de los parasitoides; aunque en el presente trabajo se colectaron un gran número de larvas frugívoras en un corto tiempo, la presencia de parasitoides fue muy baja, representando aproximadamente el 0,65\% de los individuos emergidos. Sin embargo, en condiciones naturales los índices de parasitismo de moscas de las frutas son por lo general bajos (Ovruski et al., 2000).

\section{CONCLUSIONES}

En la zona de bosque seco tropical en el Centro Universitario Regional del Norte (CURDN), municipio de Armero-Guayabal, norte del Tolima, la familia Tephritidae presenta los mayores índices de infestación y porcentajes de daño en frutos. Los lonquéidos aunque presentaron daños e índices de infestación bajos pueden contribuir al deterioro y pérdida de frutas. Especies de las familias Tephritidae y Lonchaeidae se presentan compartiendo hospederos y cohabitando en un mismo fruto. Las especies de Anastrepha cuando se encuentran en simpatría tienden a no compartir hospederos y en los casos donde sucede, no cohabitan un mismo fruto. Las especies de lonquéidos comparten hospederos y frutos, tanto con tefrítidos como entre ellas. Especies de parasitoides de la familia Braconidae, en este caso Doryctobracon areolatus, presentan una preferencia natural por larvas de moscas Tephritidae (Anastrepha spp.), mientras que especies de la Figitidae (Trybliographa nordlanderi, Lopheucoila anastrephae) prefieren larvas de las especies de Lonchaeidae (Neosilba spp.). 


\section{AGRADECIMIENTOS}

Los autores agradecen al Dr. Iain MacGowan y al Dr. Cheslavo A. Korytkowski (QEPD) por su ayuda en la determinación y confirmación de las especies de Lonchaea; a todo el personal del Herbario Toli y del Laboratorio de Dendrología "Fernando Aly Huertas Gómez" de la Universidad del Tolima, por su colaboración en la determinación de las plantas hospederas; al personal del GIMFRUT, por su apoyo en el desarrollo del proyecto. Este trabajo es el resultado del proyecto 20213, financiado por el Comité Central de Investigaciones y Desarrollo Científico de la Universidad del Tolima.

\section{REFERENCIAS BIBLIOGRÁFICAS}

ALUJA, M., RULL, J.; SIVINSKI, J.; NORRBOM, A.L.; WHARTON, R.A.; MACÍAS-ORDÓÑEZ, R.; DÍAZFLEISCHER, F.; LÓPEZ, M. 2003. Fruit flies of the genus Anastrepha (Diptera: Tephritidae) and associated native parasitoids (Hymenoptera) in the tropical rainforest biosphere reserve of Montes Azules, Chiapas, Mexico. Environ. Entomol. 32(6):1377 - 1385.

ALUJA, M.; DÍAZ-FLEISCHER, F. 2006. Foraging behavior of Anastrepha ludens, A. obliqua, and A. serpentina in response to feces extracts containing host marking pheromone. J. Chem. Ecol. 32(2):367 - 389. doi: 10.1007/s10886-005-9007-6

ALUJA, M.; SIVINSKI, J.; OVRUSKI, S.; GUILLÉN, L.; LÓPEZ, M.; CANCINO, J.; TORRES-ANAYA, A.; GALLEGOS-CHAN, G.; RUÍZ, L. 2009. Colonization and domestication of seven species of native New World hymenopterous larval-prepupal and pupal fruit fly (Diptera: Tephritidae) parasitoids. Biocontrol Sci. Tech. 19(sup1): 49 - 79. doi: 10.1080/09583150802377373.

BIRKE, A.; ALUJA, M. 2011. Anastrepha ludens and Anastrepha serpentina (Diptera: Tephritidae) do not infest Psidium guajava (Myrtaceae), but Anastrepha obliqua occasionally shares this resource with Anastrepha striata in nature. J. Econ. Entomol. 104(4):1204 - 1211. doi: 10.1603/EC11042.
BIRKE, A.; ACOSTA, E.; ALUJA, M. 2015. Limits to the host range of the highly polyphagous tephritid fruit fly Anastrepha ludens in its natural habitat. B. Entomol. Res. 105(6):743 - 753. doi: 10.1017/S0007485315000711.

BOMFIM, D.A.; GISLOTI, L.J.; UCHÔA, M.A. 2014. Fruit flies and lance flies (Diptera: Tephritoidea) and their host plants in a conservation unit of the Cerrado biome in Tocantins, Brazil. Fla. Entomol. 97(3):1139 - 1147. doi: 10.1653/024.097.0321

CANAL, N.A.; ZUCCHI, R.A. 2000. Parasitóides: Braconidae. pp. 119 - 126. En: Malavasi, A.; Zucchi, R.A. (eds.). Moscas-das-frutas de importância econômica no Brasil: conhecimento básico e aplicado. Holos Editora. Ribeirão Preto, Brasil. 325p.

CARREJO, N.S.; GONZÁLEZ, R. 1999. Parasitoids reared from species of Anastrepha (Diptera: Tephritidae) in Valle del Cauca, Colombia. Fla. Entomol. 82(1):113 118.

CASTAÑEDA, M.R.; OSORIO, A.; CANAL, N.A.; GALEANO, P.E. 2010. Especies, distribución y hospederos del género Anastrepha Schiner en el departamento del Tolima, Colombia. Agron. Colomb. 28(2):265 - 271.

DELGADO, A.; IMBACHI, K.; KONDO, T. 2010a. Reporte de una mosca del género Neosilba McAlpine (Diptera: Lonchaeidae) asociada a la pudrición basal del fruto de la pitaya amarilla, Selenicereus megalanthus (K. Schum. ex Vaupel) Moran en Colombia. Bol. Mus. Ent. Univ. Valle. 11(1):31 - 33.

DELGADO, A.; KONDO, T.; IMBACHI, K.; QUINTERO, E.M.; MANRIQUE, M.B.; MEDINA, J.A. 2010b. Biología y algunos datos morfológicos de la mosca del botón floral de la pitaya amarilla, Dasiops saltans (Townsend) (Diptera: Lonchaeidae) en el Valle del Cauca, Colombia. Bol. Mus. Ent. Univ. Valle. 11(2):1 - 10.

DEVESCOVI, F.; LIEND0, M.C.; BACHMANN, G.E.; BOUVET, J.P.; MILLA, F.H.; VERA, M.T.; CLADERA, J.L.; SEGURA, D.F. 2015. Fruit infestation patterns by Anastrepha fraterculus and Ceratitis capitata reveal that cross-recognition does not lead to complete avoidance 
of interspecific competition in nature. Agr. Forest Entomol. 17(3):325 - 335. doi: 10.1111/afe.12111.

DÍAZ-FLEISCHER, F.; PAPAJ, D.R.; PROKOPY, R.J. NORRBOM, A.L.; ALUJA, M. 2000. Evolution of fruit fly oviposition behavior. pp. 811 - 481. En: Aluja, M.; Norrbom, A.L. (eds.). Fruit Flies (Tephritidae): Phylogeny and Evolution of Behavior. CRC Press. Boca Raton, Florida. 984 p.

DUYCK, P.F.; DAVID, P.; QUILICI, S. 2004. A review of relationships between interspecific competition and invasions in fruit flies (Diptera: Tephritidae). Ecol. Entomol. 29(5):511 - 520. doi: 10.1111/j.03076946.2004.00638.x

GALEANO-OLAYA, P. E.; CANAL, N.A. 2012. New species of Neosilba McAlpine (Diptera: Lonchaeidae) and new records from Colombia. Pap. Avulsos Zool. 52(31):361 $-385$.

GARCIA, F.R.M.; RICALDE, M.P. 2013. Augmentative biological control using parasitoids for fruit fly management in Brazil. Insects. 4(1):55 - 70. doi:10.3390/insects4010055.

GUIMARÃES, J.A.; DIAZ, N.B.; ZUCCHI, R.A. 2000. Parasitóides - Figitidae (Eucoilinae). pp. 127 - 134. En: Malavasi, A.; Zucchi, R.A. (eds.). Moscas-das-frutas de importância econômica no Brasil: conhecimento básico e aplicado. Holos Editora. Ribeirão Preto, Brasil. 325p.

GONÇALVES, R.S.; ANDREAZZA, F.; LISBÔA, H.; GRÜTZMACHER, A.D.; VALGAS, R.A.; MANICA-BERTO, R.; NÖRNBERG, S.D.; NAVA, D.E. 2016. Basis for the development of a rearing technique of Aganaspis pelleranoi (Hymenoptera: Figitidae) in Anastrepha fraterculus (Tephritidae: Diptera). J. Econ. Entomol. 109(3):1094 - 1101. doi:10.1093/jee/tow069.

GONZÁLEZ, R.; CARREJO; N.S. 1993. Cohabitación por dos especies de Anastrepha (Diptera: Tephritidae) en frutos de caimito, Pouteria caimito (Ruiz y Pavón) Radlkofer. Bol. Mus. Ent. Univ. Valle. 1(2):13 - 21.

KORYTKOWSKI, C.A.; OJEDA, D. 1971. Revisión de las especies de la familia Lonchaeidae en el Perú (Díptera: Acalyptratae). Rev. Peru Entomol. 14(1):87 - 116.
KORYTKOWSKI, C.A. 2008. Manual para la identificación de moscas de la fruta Género Anastrepha Schiner, 1868. Octava edición. Vicerrectoría de Investigación y Postgrado, Programa de Maestría en Entomología, Universidad de Panamá, Ciudad de Panamá. 145p.

LIENDO, M.C.; DEVESCOVI, F.; BOCA, T.; CLADERA, J.L.; VERA, M.T.; SEGURA, D.F. 2016. Patterns of resource distribution among conspecific larvae in two fruit fly species: Anastrepha fraterculus and Ceratitis capitata (Diptera: Tephritidae). Agr. Forest Entomol. 18:349 356. doi:10.1111/afe.12166.

LOPES, G.N.; SOUZA-FILHO, M.F.; GOTELLI, N.J.; LEMOS, L.J.U.; GODOY, W.A.C.; ZUCCHI, R.A. 2015. Temporal overlap and co-occurrence in a guild of subtropical tephritid fruit flies. PLoS ONE. 10(7):1 - 14. doi:10.1371/journal.pone.0132124.

MACGOWAN, I.; OKAMOTO, T. 2013. New species of Lonchaeidae (Diptera: Schizophora) from Japan and a re-evaluation of genus Setisquamalonchaea Morge. Entomol. Sci. 16(2):196 - 202. doi:10.1111/ens.12003.

MCALPINE, J.F. 1987. Lonchaeidae, pp. 791 - 797. En: McAlpine, J.F. (ed.). Manual of Nearctic Diptera. Volume 2. Canada Communication Group - Publishing. Ottawa, Canada. 1332p.

MCALPINE, J.F.; STEYSKAL, G.C. 1982. A revision of Neosilba McAlpine with a key to the world genera of Lonchaeidae (Diptera). Can. Entomol. 114(2):105 - 137.

MEDINA, J.A.; KONDO, T. 2012. Listado taxonómico de organismos que afectan la pitaya amarilla, Selenicereus megalanthus (K. Schum. ex Vaupel) Moran (Cactaceae) en Colombia. Corpoica Cienc. Tecnol. Agropecu. 13(1):41 - 46.

MIRANDA, D. 2011. Estado actual de fruticultura colombiana y perspectivas para su desarrollo. Rev. Bras. Frutic. 33(spe1):199 - 205.

NICÁCIO, J.; UCHÔA, M.A. 2011. Diversity of frugivorous flies (Diptera: Tephritidae and Lonchaeidae) and their relationship with host plants (Angiospermae) in environments of South Pantanal Region, Brazil. Fla. Entomol. 94(3):443 - 466. doi: 10.1653/024.094.0309. 
NICÁCIO, J.N.; UCHÔA, M.A.; FACCENDA, O.; GUIMARÃES, J.A.; MARINHO, C.F. 2011. Native larval parasitoids (Hymenoptera) of frugivorous Tephritoidea (Diptera) in South Pantanal Region, Brazil. Fla. Entomol. 94(3):407 - 419. doi: 10.1653/024.094.0305.

NORRBOM, A.L.; KORYTKOWSKI, C.A.; ZUCCHI, R.A.; URAMOTO, K.; VENABLE, G.L.; MCCORMICK, J.; DALLWITZ, M.J. 2012. Anastrepha and Toxotrypana: descriptions, illustrations, and interactive keys. En: DELTA - Description Language for Taxonomy. http:// delta-intkey.com/anatox/; consulta: septiembre, 2014.

NÚÑEZ, L.; GÓMEZ, R.; GUARÍN, G.; LEÓN, G. 2004a. Moscas de las frutas (Díptera: Tephritidae) y parasitoides asociados con Psidium guajava L. y Coffea arabica L. en tres municipios de la Provincia de Vélez (Santander, Colombia) Parte 1: Índices de infestación y daño por moscas de las frutas (Díptera: Tephritidae). Corpoica Cienc. Tecnol. Agropecu. 5(1):5 - 12.

NÚÑEZ, L.; GÓMEZ, R.; GUARÍN, G.; LEÓN, G. 2004b. Moscas de las frutas (Díptera: Tephritidae) y parasitoides asociados con Psidium guajava L. y Coffea arabica L. en tres municipios de la Provincia de Vélez (Santander, Colombia) Parte 2: Identificación y evaluación de parasitoides del orden Hymenoptera. Corpoica Cienc. Tecnol. Agropecu. 5(1):13 - 21.

OVRUSKI, S.; ALUJA, M.; SIVINSKI, J.; WHARTON, R. 2000. Hymenopteran parasitoids on fruit-infesting Tephritidae (Diptera) in Latin America and the southern United States: diversity, distribution, taxonomic status and their use in fruit fly biological control. Integr. Pest Manag. Rev. 5(2):81 - 107.

PORTILLA, M.; GONZÁLEZ, G.; NÚÑEZ, L. 1994. Infestación, reconocimiento e identificación de moscas de las frutas y de sus enemigos naturales en café. Rev. Colomb. Entomol. 20(4):261 - 266.

QUINTERO, E.M.; LÓPEZ, I.C.; KONDO, T. 2012. Manejo integrado de plagas como estrategia para el control de la mosca del botón floral del maracuyá Dasiops inedulis Steyskal (Diptera: Lonchaeidae). Corpoica Cienc. Tecnol. Agropecu. 13(1):31 - 40.
RUIZ-HURTADO, F.M.; RAMIREZ-QUIMBAYO, J.H.; ROJAS-PLAZAS, B.; GALEANO-OLAYA, P.E.; CANAL, N.A. 2013. Diversidad de parasitoides (Hymenoptera) de moscas frugívoras (Díptera: Tephritoidea) en dos áreas cafeteras del departamento del Tolima, Colombia. Rev. Tumbaga. 2(8):29 - 53.

SANTAMARÍA, M.Y.; CASTRO, A.P.; EBRATT, E.E.; BROCHERO, H.L.M. 2014. Caracterización de daños de moscas del género Dasiops (Diptera: Lonchaeidae) en Passiflora spp. (Passifloraceae) cultivadas en Colombia. Rev. Fac. Nal. Agr. Medellín. 67(1):7151 - 7162.

SARMIENTO, C.E.; AGUIRRE, H.; MARTÍNEZ, A., J. 2012. Anastrepha (Diptera: Tephritidae) y sus asociados: dinámica de emergencia de sus parasitoides en frutos de tres especies de plantas. Bol. Mus. Ent. Univ. Valle. 13(1):25 - 32.

SEGURA, D.F.; VERA, M.T., CAGNOTTI, C.L.; VACCARO, N.; COLL, O.; OVRUSKI, S.M.; CLADERA, J.L. 2006. Relative abundance of Ceratitis capitata and Anastrepha fraterculus (Diptera: Tephritidae) in diverse host species and localities of Argentina. Ann. Entomol. Soc. Am. 99(1):70 - 83. doi: 10.1603/0013-8746(2006)099[0070:RAOCCA]2.0. $\mathrm{CO} ; 2$.

SILVA, M.A.; BEZERRA, G.C.D.; MASTRANGELO, T. 2012. The host marking pheromone application on the management of fruit flies - a review. Braz. Arch. Biol. Technol. 55(6):835 - 842. doi: 10.1590/S151689132012000600005.

SIVINSKI, J.; ALUJA, M.; PIÑERO, J.; OJEDA, M. 2004. Novel analysis of spatial and temporal patterns of resource use in a group of tephritid flies of the genus Anastrepha. Ann. Entomol. Soc. Am. 97(3):504 - 512. doi: 10.1603/0013-8746(2004)097[0504:NAOSAT]2. $0 . \mathrm{CO} ; 2$.

UCHÔA, M.A. 2012. Fruit flies (Diptera: Tephritoidea): biology, host plants, natural enemies, and the implications to their natural control. pp. 271-300. En: Larramendy, M.L. y Soloneski, S. (eds.). Integrated Pest Management and Pest Control - Current and Future Tactics. InTech. Rijeka, Croacia. 682 p. doi: 10.5772/31613. 
UCHÔA-FERNANDES, M.A.; ZUCCHI, R.A. 1999. Metodología de colecta de Tephritidae y Lonchaeidae frugívoros (Diptera: Tephritoidea) y sus parasitoides (Hymenoptera). An. Soc. Entomol. Bras. 28(4):601 610.

UCHÔA-FERNANDES, M.A.; MOLINA, R.M.S.; OLIVEIRA, I.; ZUCCHI, R.A.; CANAL, N.A.; DÍAZ, N.B. 2003. Larval endoparasitoids (Hymenoptera) of frugivorous flies (Diptera, Tephritoidea) reared from fruits of the cerrado of the State of Mato Grosso do Sul, Brazil. Rev. Bras. Entomol. 47(2):181 - 186.

UNIVERSIDAD DEL TOLIMA. 2012. Granja de Armero Centro Universitario Regional del Norte - C.U.R.D.N. En: UT, http://www.ut.edu.co/academi/institucional1/ facultades-e-instituto-de-educacion-a-distancia/ facultad-de-ingenieria-agronomica/servicios-yproductos/granjas/centro-universitario-regional-delnorte; consulta: junio, 2014.

WHARTON, R.A.; YODER, M.J. 2014. Parasitoids of fruit-infesting Tephritidae. En: Parasitic Hymenoptera Research Labs - Texas A\&M University, http://paroffit. org/public/site/paroffit/home; consulta: agosto, 2014.

WYCKHUYS, K.A.G.; KORYTKOWSKI, C.; MARTINEZ, J.; HERRERA, B.; ROJAS, M.; OCAMPO, J. 2012. Species composition and seasonal occurrence of Diptera associated with passionfruit crops in Colombia. Crop Prot. 32:90 - 98. doi:10.1016/j.cropro.2011.10.003.

YEPES, F.; VÉLEZ, R. 1989. Contribución al conocimiento de las moscas de las frutas (Tephritidae) y sus parasitoides en el departamento de Antioquia. Rev. Fac. Nal. Agr. Medellín. 42(2):73 - 98. 